\title{
Rapport mondial des Nations Unies sur la mise en valeur des ressources en eau 2020 L'eau et les changements climatiques
}

\author{
Daniel Loudière $^{*}$,a et Philippe Gourbesville ${ }^{1}$ \\ ${ }^{1}$ Université de Nice, Nice, France
}

Reçu le 5 mai 2020 / Accepté le 11 mai 2020

\begin{abstract}
Résumé - Le rapport mondial des Nations Unies sur la mise en valeur des ressources en eau 2020 est le fruit d'une collaboration entre agences des Nations Unies sous pilotage UNESCO et UN Water. Cette année l'accent a été porté sur la disponibilité des ressources en eau, les effets des changements climatiques (CC) sur le cycle de l'eau, l'atténuation des CC et l'adaptation pour ce qui relève du secteur de l'eau. Le contexte international (conventions, traités, etc.) est présenté, avec une attention particulière au plan de développement durable 2030 et les Objectifs du Développement Durable, notamment l'ODD 6 relatif à l'accès à l'eau et à l'assainissement.
\end{abstract}

Mots clés : Rapport mondial / Nations Unies / eau / changements climatiques / atténuation / adaptation

Abstract - World Water Development Report-Water and Climate Change. The 2020 World Water Report is published by UNO group, under the coordination of UNESCO and UN Water. For this report, the following issues have been developed: availability of water resources, impacts of climate change (CC) on water cycle, mitigation and adaptation to $\mathrm{CC}$ in the water sector. The international context has been described (conventions, treaties, etc.), with a specific care to the Sustainable Development Plan 2030 and the Sustainable Development Goals, specially the SDG 6 devoted to the access to water and sanitation.

Keywords: World Report / United Nations / water / climate change / mitigation / adaptation

\section{Introduction}

Le rapport publié le 22 mars 2020 à l'occasion de la journée mondiale de l'eau porte plus particulièrement sur l'articulation eau et changements climatiques. La rédaction a été pilotée par l'UNESCO et UN Water (secrétariat permanent pour l'eau de l'ONU) avec les contributions de nombreuses agences de l'ONU (FAO, OMS, OMM, ONU-Habitat, UNIDO, etc.) ainsi que de la Banque Mondiale. Pour mémoire, on peut rappeler qu'une vingtaine d'agences des Nations Unies sont intéressées par les milieux aquatiques, les ressources en eau et leurs usages. Le rapport est disponible ainsi que son résumé sur les sites de l'UNESCO et d'UN-Water (UNESCO et ONU-Eau, 2020).

Cet article de synthèse aborde les points essentiels qui sont largement développés dans 259 pages du rapport original.

\footnotetext{
*Correspondance : daniel.loudiere@free.fr

${ }^{a}$ SHF Président honoraire.
}

\section{L'évolution des usages de l'eau}

La FAO, à travers le service Aquastat, s'efforce de suivre les principales données du grand cycle de l'eau douce par cumul des données issues des pays. Le volume annuel des précipitations sur ces pays est de l'ordre de $110000 \mathrm{~km}^{3}: 56 \%$ repart en évapotranspiration (forêts et espaces naturels), $5 \%$ pour l'agriculture pluviale et $39 \%$ en ruissellement et infiltration pour alimenter les aquifères et les rivières. Pour ce qui relève des prélèvements, le graphique ci-dessous montre une croissance lente avant 1940 et une croissance forte entre 1950 et 1980 pour atteindre $4300 \mathrm{~km}^{3}$ en 2010 . L'évaporation à partir des lacs artificiels apparaît dans ce bilan. Dans la même période 1900-2010, la population du monde passait de 1,7 à 6,8 milliards d'habitants n'expliquant que partiellement l'évolution globale des prélèvements. En 2010, les prélèvements pour l'agriculture irriguée représentent environ les $2 / 3$ du total (Fig. 1)

Les chiffres globaux sont marqués par les données issues de pays à forte hydraulicité et cachent des disparités énormes 


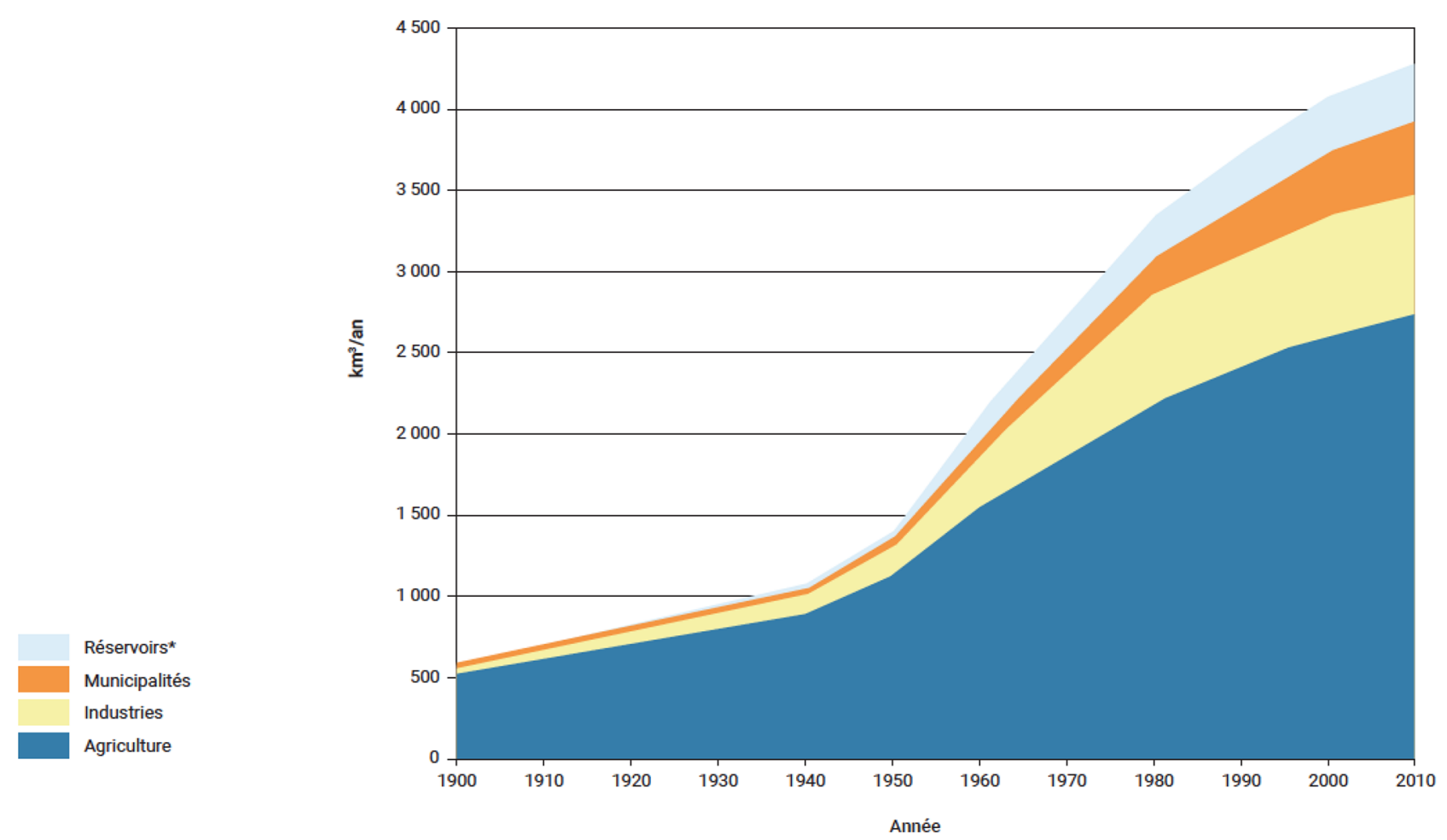

* Évaporation à partir de lacs artificiels.

Fig. 1. Taux mondial de prélèvements d'eau au cours du $20^{\mathrm{e}}$ siècle, en $\mathrm{km}^{3} /$ an (Aquastat, 2010).

expliquant qu'actuellement 2,2 milliards de personnes ne disposent pas d'un accès à l'eau potable et que 4,2 milliards - soit plus de la moitié de la population mondiale - sont privées de systèmes d'assainissement.

\section{Les menaces les plus alarmantes}

Bien que les menaces soient traitées à des endroits divers du rapport, celui-ci fait clairement apparaître :

- La baisse de qualité des milieux aquatiques : augmentation de la température de l'eau, diminution de l'oxygène dissous, croissance du taux de polluants d'origine agricole ou pharmaceutique et réduction des débits en étiage;

- Les atteintes à la santé des populations, la persistance des maladies hydriques;

- La perte de biodiversité à travers la disparition ou la dégradation de nombreux écosystèmes, notamment des zones humides.

Par ailleurs le rapport fait le lien avec d'autres politiques publiques tout aussi fondamentales : la sécurité alimentaire, les établissements urbains et ruraux, la production d'énergie et plus globalement le développement durable.

\section{Les changements climatiques}

Le prologue et le chapitre 1 du rapport traitent essentiellement des changements climatiques en s'appuyant sur les travaux du GIEC (Rapports du GIEC). L'influence humaine sur le système climatique, le rôle des gaz à effet de serre (GES) dans le réchauffement climatique, le réchauffement général de la terre et la surélévation du niveau des océans bénéficient désormais d'un consensus scientifique fort.

La qualification des changements hydrologiques provenant des changements climatiques est un art complexe: les tendances en matière de précipitations annuelles moyennes sont incertaines dans de nombreuses régions. Selon le rapport, les modèles climatiques mondiaux s'accordent dans une vaste mesure sur l'augmentation du nombre d'évènements météorologiques extrêmes (Fig. 2). Les précipitations extrêmes devenant plus intenses et plus fréquentes devraient accroitre les risques d'inondation; les vagues de chaleur surviendront plus souvent et dureront plus longtemps, rendant les sécheresses plus intenses (Fig. 3).

Notons que pour ce qui relève des crues, les effets des changements climatiques donnent lieu à des interprétations différenciées (Blöschl et al., 2019); par contre, l'augmentation de la vulnérabilité a clairement un impact majeur sur les coûts des inondations catastrophiques.

Les processus hydrologiques devraient également être altérés, avec des effets contradictoires sur la disponibilité des ressources en eau. Selon les régions, les précipitations annuelles moyennes augmenteront ou diminueront; le pourtour méditerranéen serait particulièrement concerné par la décroissance des précipitations. L'évapotranspiration réelle pourrait croître notablement dans certains bassins versants, les contrastes pluviométriques entre saison des pluies et saison sèche seraient encore plus marqués, la contribution en saison sèche de la fonte des glaces et de la neige aux débits des 


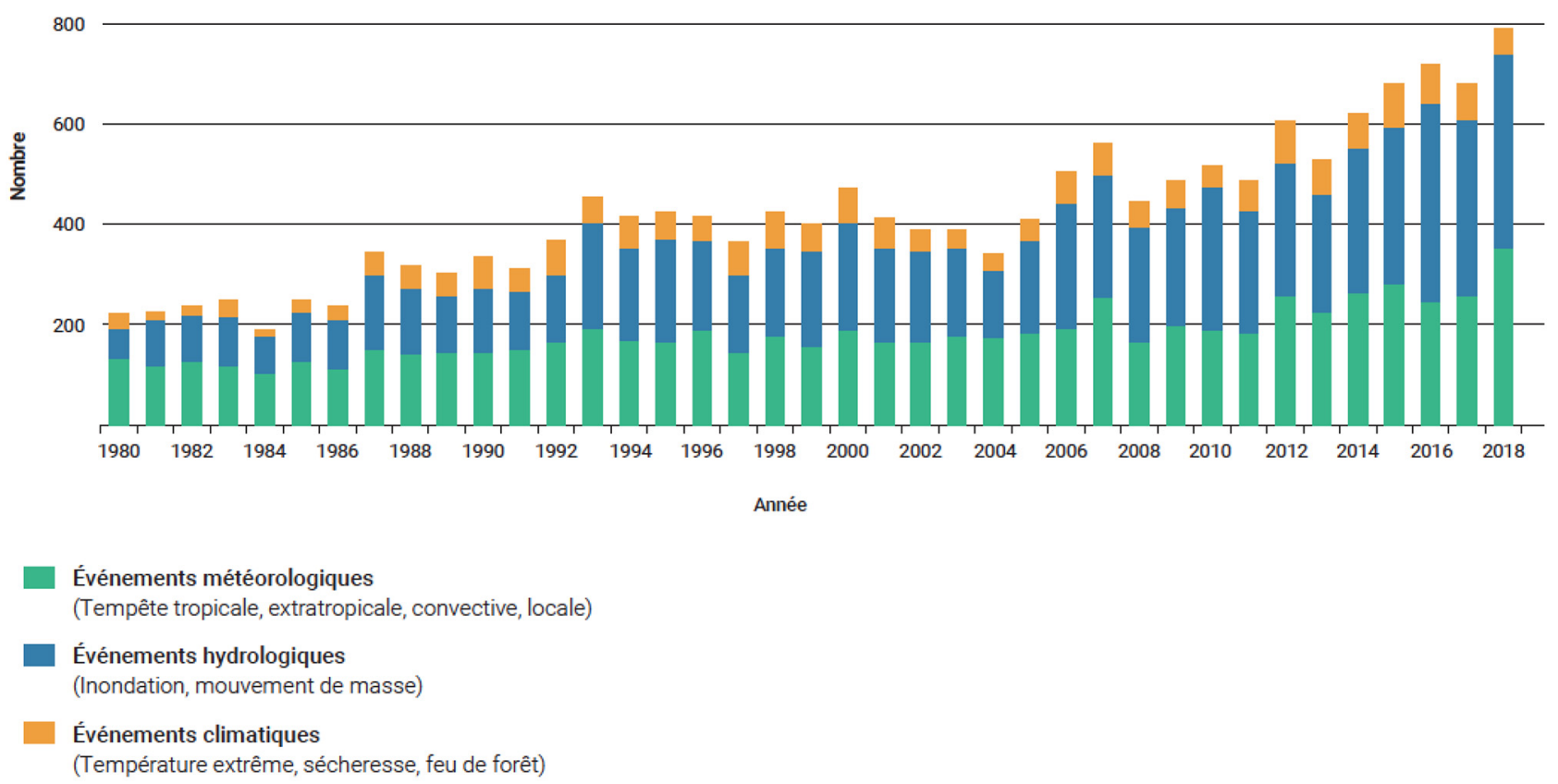

Note : Les événements attribués ont entraîné au moins un décès ou engendré des pertes normalisées équivalentes ou supérieures à 100 000, 300 000, 1 million ou 3 millions de dollars EU (selon le classement de la Banque mondiale du groupe de revenu du pays touché.

Fig. 2. Catastrophes naturelles mondiales liées au climat, par risque et par nombre d'événements pertinents, $1980-2018$ (Munich et NatCatService, 2019).

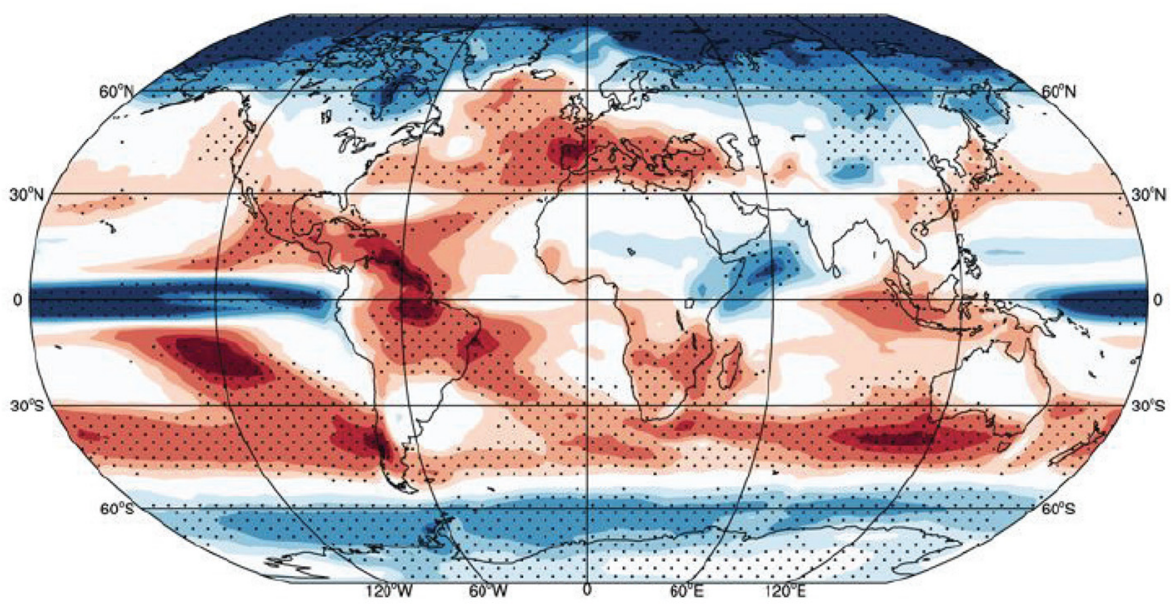

(jours/an)

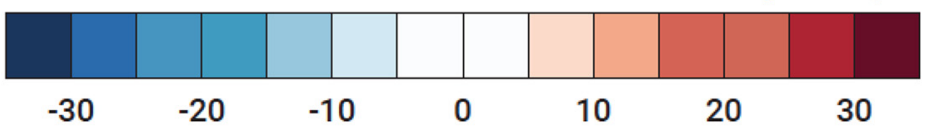

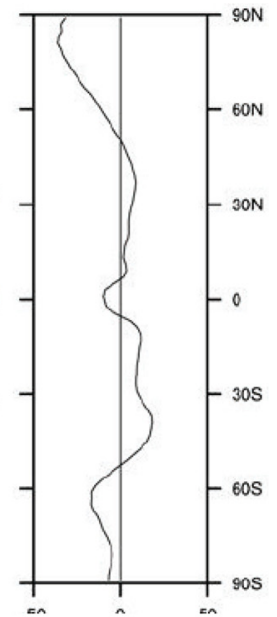
valeurs moyennes par zone.

Fig. 3. Changement dans la moyenne annuelle et la fréquence des jours secs (jours/an) d'ici 2060-2089 par rapport à la période historique 19601989, fondées sur l'ensemble de modèles CMIP5 en utilisant le scénario RCP8.5 (Polade et al., 2014 ; Fig. 3). 
rivières se réduirait. L'impact direct sur le budget pour l'eau terrestre sera très fort dans certaines régions du monde, notamment au regard des débits d'étiage et de la sécheresse des sols.

\section{Les régions les plus concernées}

Le rapport insiste sur les petits États insulaires en développement (PEID), les régions semi-arides, les arrièrepays littoraux et les zones montagneuses. Les PEID sont concernés par l'élévation du niveau de la mer, le déplacement de l'interface entre eau de mer et eau douce vers l'intérieur des pays et surtout une diminution de l'approvisionnement en eau en conséquence de précipitations réduites. Ces phénomènes sont exacerbés par une demande en eau fortement croissante liée au tourisme et au développement économique.

Les régions semi-arides sont généralement définies comme des régions dans lesquelles l'évaporation potentielle (ETP) dépasse largement la précipitation annuelle. Le risque de dégradation des terres et la désertification sont les conséquences logiques d'une aridité accrue et d'un réchauffement accéléré, amplifiés par une forte croissance démographique. L'Afrique du Sud et l'Asie méridionale sont cités. Néanmoins, le pourtour méditerranéen et le Moyen Orient ne sont pas particulièrement mis en exergue malgré les enjeux. La situation en matière de précipitations s'annonce catastrophique en Afrique du Nord selon plusieurs scénarios.

Les arrière-pays littoraux, zones côtières de faible altitude, sont sensibles à l'élévation du niveau des mers, aux débits de crues des fleuves ainsi qu'aux ondes de tempêtes et à l'intensité des vents. Plus de 600 millions de personnes vivent dans ces zones menacées. Quel avenir pour les deltas majeurs et densément peuplés du Nil ou du Mékong où s'ajoutent aux multiples usages les phénomènes géologiques et sédimentaires ?

Les régions montagneuses de haute altitude subissent un réchauffement accéléré dont se ressentent les glaciers et les sommets enneigés. La perturbation sur l'hydraulicité des rivières touche aussi bien les bassins amont à relief accidenté que les zones aval en plaine.

\section{L'eau et l'atténuation des changements climatiques (CC)}

L'atténuation consiste en des interventions humaines visant à réduire les sources de gaz à effet de serre ou à renforcer les puits de gaz à effet de serre (GES). Bien que des options d'atténuation soient également disponibles dans tous les secteurs majeurs liés à l'eau, elles demeurent largement ignorées.

Les services d'eau potable et d'assainissement seraient responsables de 3 à $7 \%$ des émissions de GES, mais auxquels s'ajoutent les émissions associées au rejet d'eaux usées non traitées (80 à $90 \%$ des eaux usées). Le secteur représente environ $4 \%$ de la consommation d'électricité dans le monde, proportion en forte croissance potentielle avec le développement du dessalement de l'eau de mer et les progrès attendus en matière d'accès à l'eau potable et à l'assainissement (Objectif du Développement Durable 6/ODD 6). Parallèlement le secteur de l'eau pourrait réduire sa consommation d'énergie de $15 \%$ d'ici 2040.
Les réservoirs des barrages sont des sources d'émission de $\mathrm{CH}^{4}$ avec la décomposition des matières organiques stockées dans les retenues (soit environ 1,5\% des émissions d'équivalent en $\mathrm{CO}^{2}$ ). Ce sujet est un élément important à prendre en compte pour les maîtres d'ouvrages des barrages en projet.

Les zones humides, y compris les tourbières, stockeraient deux fois plus de carbone que les forêts. La dégradation des tourbières ou leur drainage à des fins agricoles sont autant d'actions contraires à ce grand objectif de décarbonation des activités économiques, en plus du maintien de la biodiversité.

Le rôle essentiel des forêts et des sols agricoles pour le stockage du carbone est aussi évoqué dans le rapport.

\section{L'eau et l'adaptation aux changements climatiques}

L'adaptation associe les options naturelles, construites et technologiques, à des mesures sociales et institutionnelles visant à réduire les risques ou à exploiter tout avantage éventuel découlant des changements climatiques. Des options d'adaptation existent dans tous les secteurs liés à l'eau et devraient être étudiées et mises en œuvre lorsque possible.

L'approche retenue est clairement celle de la Gestion Intégrée des Ressources en Eau (GIRE) et ses multiples composantes : connaissance des ressources, des usages et des besoins, information et concertation avec les parties prenantes, planification avec tous les outils d'une mise en œuvre optimisée tant en termes de gouvernance que de financement. Deux domaines ont été traités de façon approfondie dans le rapport: l'irrigation et l'eau potable des villes.

Les mesures pratiques de gestion de l'eau destinée à l'agriculture ont été placées sous le chapeau d'une Agriculture Intelligente Concertée (AIC) succédant à l'agriculture de précision et à l'agriculture fondée sur la nature. Les réponses de l'AIC en matière de mobilisation des ressources en eau sont décrites comme suit, au niveau local:

- Bénéficier des progrès de la météorologie: prévisions météorologiques, conseil en agriculture, assurance météorologique ;

- Mobilisation raisonnée de l'eau : recharge des aquifères par des pratiques agricoles favorisant l'infiltration, collecte et valorisation des eaux usées, gestion communautaire des eaux, utilisation de pompes solaires;

- Choix optimal des espèces et des semences: variétés végétales et espèces adaptées, création de banques de semences ;

- Stockage du carbone et priorité aux nutriments naturels : agroforesterie, travail minimal du sol, assolements adaptés, gestion du bétail, gestion intégrée des nutriments et développement des biocarburants ;

- Cadre institutionnel et mise en marché structurés: liens intersectoriels, institutions locales, stratégie pour l'égalité des genres, planification des interventions d'urgence, services financiers, informations commerciales et gestion des risques hors des exploitations agricoles.

Le rapport contient des développements intéressants sur l'irrigation déficitaire contrôlée (souvent dénommée irrigation de complément) en comparaison de l'agriculture pluviale 
non-irriguée et de l'irrigation intégrale «très » consommatrice en eau.

En matière d'eaux urbaines, un besoin accru de résilience est souligné. Les agglomérations abriteront près de $70 \%$ de la population mondiale d'ici 2050. D'ici 2030, le monde devrait compter 43 mégapoles de plus de 10 millions d'habitants, dont la majorité se trouvera dans des régions en développement. De nombreuses villes ont souffert, au cours de la dernière décennie, de problèmes de ressources en eau. La bonne compréhension du développement urbain, la planification des infrastructures physiques et institutionnelles, l'attention à porter à la résilience en réponse aux crises inévitables et enfin l'inscription de la gestion de l'eau dans un contexte plus large (géographique et sectoriel) apparaissent comme indispensables pour éviter des catastrophes majeures. Entre les solutions locales comme la gestion de la demande en eau, souvent efficace pour atténuer la pénurie, et les transferts à longue distance, une vaste panoplie de solutions existe sans que soient mises en avant des méthodes prescriptives permettant d'aborder en toute efficience la résilience des ressources en eau en milieu urbain.

\section{Innovations technologiques et connaissances des citoyens}

Cet avant-dernier chapitre du rapport est au cœur des préoccupations de la SHF et met en évidence les défis et les possibilités que présente la promotion de la recherche et de l'innovation pour appuyer la prise de décisions éclairées.

La priorité est d'accélérer la mise en œuvre dans tous les pays des connaissances et des technologies disponibles, mais également de promouvoir la création de nouveaux outils et de nouvelles approches par le biais d'actions de recherchedéveloppement. Les décideurs politiques et les scientifiques sont confrontés à la difficulté d'évaluer et d'anticiper les changements attendus et leurs conséquences potentielles. Les incertitudes des modèles, notamment climatiques et hydrologiques, ne sauraient durablement servir de prétexte à l'inaction face aux CC. Les approches attendues sont multidisciplinaires, intersectorielles et multidimensionnelles et force est de reconnaître que peu de programmes de développement ont ces ambitions.

L'innovation technologique attendue doit s'appliquer aux processus climatiques et hydrologiques, à la gestion des ressources en eau (récupération et réutilisation), à l'adaptation des infrastructures, à la réduction des coûts (traitement, distribution et épuration), à l'efficacité et à la sobriété des usages et enfin à l'accès du plus grand nombre à l'eau potable et à l'assainissement.

Parmi les innovations technologiques les plus prometteuses, le rapport cite :

- La télédétection satellitaire avec l'apparition de capteurs plus performants ;

- Les Technologies de l'Information et de la Communication au sens large, la collecte massive de données et leur analyse, l'apport des techniques de l'Intelligence Artificielle.

Le fossé trop marqué entre scientifiques et décideurs politiques freine la mise en œuvre d'innovations. L'information sur ces innovations et les incertitudes associées doit être largement disponible et formulée de façon claire et simple pour promouvoir des responsabilités assumées.

Sous le vocable des sciences participatives, le rapport prône le développement de :

- La participation de la société civile à la collecte et à la fourniture des informations relatives à l'eau;

- La formation et la sensibilisation des citoyens aux défis majeurs du secteur de l'eau, citoyens devenant acteurs de l'atténuation et de l'adaptation aux CC.

\section{Quelques commentaires en conclusion}

Les liens entre les CC et l'eau ont été traités de façon assez complète pour ce qui relève de l'atténuation, du plan de développement durable 2030 ainsi que des objectifs du développement durable, notamment de l'objectif de développement durable 6 relatif à l'accès à l'eau et à l'assainissement.

Comme pour tout rapport international, les cadres politiques internationaux et les principaux accords relatifs à l'eau sont effectivement présentés.

Les rapports de l'eau avec la préservation de la santé humaine, avec l'alimentation et les secteurs de l'énergie et de l'industrie font l'objet d'analyses intéressantes.

Lorsque les auteurs du rapport traitent des évènements extrêmes liés à l'eau et de la gestion des risques, ils évoquent principalement les risques d'inondations alors que les sécheresses risquent à terme, compte-tenu des mesures d'atténuation des CC bien modestes en regard des enjeux, de devenir un sujet de tensions et de crises majeures dans plusieurs régions du monde.

Des perspectives régionales ont été présentées et ont été élaborées au niveau de cinq très grandes régions gardant un caractère général. Cette approche a le défaut de ne pas mettre en exergue les situations catastrophiques de stress hydrique qui frappera dès les années 2050 certains pays dont la situation en regard des ressources en eau est déjà très difficile.

Enfin le rapport traite de la gouvernance de l'eau pour la résilience nécessaire pour faire face aux changements climatiques (y compris par la lutte contre la pauvreté et les inégalités), et présente des considérations économiques et financières.

Le rapport se termine par un appel à l'action urgente et à «aller de l'avant». Cet appel à l'action fait suite à deux décennies de rapports similaires alors que les incertitudes se réduisent et que les faits alarmants se multiplient. Avec cette synthèse, les auteurs souhaitent contribuer à crédibiliser la dernière déclaration du rapport: " À une époque caractérisée par une multitude d'études et d'articles sur les changements climatiques et d'autres crises environnementales mondiales, et à la lumière des revers perçus lors de la réunion de la COP 25 , ce rapport propose une série de réponses pratiques, en termes de politique, de financement et d'action sur le terrain, pour soutenir nos objectifs collectifs et nos aspirations individuelles à réaliser un monde durable et prospère pour tous ».

\section{Références}

Aquastat. 2010. Global Water Withdrawal. Site Web d'AQUASTAT. Rome: Organisation des Nations Unies pour l'alimentation et l'agriculture (FAO). www.fao.org/nr/water/aquastat/water_use/ image/WithTimeNoEvap_eng.pdf. 
Blöschl G, et al. 2019. "Changing climate both increases and decreases European river floods". Nature 573. https://doi.org/ 10.1038/s4586-019-1495-6.

Munich RE, NatCatService. 2019. NatCatService. Natural catastrophe statistics online. Site Web de Munich. www.munichre.com/ en/reinsurance/business/non-life/natcatservice/index.html.

Polade SD, Pierce DW, Cayan DR, Gershunov A, Dettinger MD. 2014. "The key role of dry days in changing regional climate and precipitation regimes". Sci Rep 4(4364): 1-8. https://doi.org/ 10.1038/srep04364.

Rapports du GIEC. https://www.ipcc.ch/languages-2/francais/publi cations/.

UNESCO, ONU-Eau. 2020. Rapport mondial des Nations Unies sur la mise en valeur des ressources en eau 2020: l'eau et les changements climatiques. Paris: UNESCO. www.unesco.org/ wwap/wwdr/2020.

Citation de l'article : Loudière D, Gourbesville P. 2020. Rapport mondial des Nations Unies sur la mise en valeur des ressources en eau 2020. La Houille Blanche : 76-81 